\title{
Closing the Brief Case: Postinfectious Glomerulonephritis as an Unexpected Sequela of Drinking Raw Milk
}

\author{
M. Suzanne Whitworth, ${ }^{\text {a }}$ (D) Morgan A. Pence ${ }^{\text {b }}$ \\ aDepartment of Infectious Diseases, Cook Children's Medical Center, Fort Worth, Texas, USA \\ bDepartments of Laboratory and Pathology, Cook Children's Medical Center, Fort Worth, Texas, USA
}

KEYWORDS Campylobacter, glomerulonephritis

\section{ANSWERS TO SELF-ASSESSMENT QUESTIONS}

1. Which of the following is a risk factor for campylobacteriosis?
a. Camping
b. Swimming in a chlorine-treated pool
c. Ingestion of unpasteurized milk
d. Ingestion of fried chicken

Answer: c. Ingestion of unpasteurized/raw milk is a risk factor for campylobacteriosis. Camping and swimming in adequately chlorinated pools are not risk factors, but ingestion of untreated or inadequately treated water during these events would be a risk factor. Ingestion of fried chicken, assuming it is properly cooked, is not a risk factor. However, ingestion of undercooked poultry is a risk factor.

2. Most Campylobacter selective agars are selective only for which two species?
a. Campylobacter jejuni and Campylobacter coli
b. Campylobacter coli and Campylobacter lari
c. Campylobacter jejuni and Campylobacter lari
d. Campylobacter jejuni and Campylobacter upsaliensis

Answer: a. Cefoperazone, a cephalosporin antibiotic found in most Campylobacter selective agars, is inhibitory to most Campylobacter spp. The majority of strains of $C$. jejuni and $C$. coli are resistant to cefoperazone and will grow on selective media; however, susceptible strains have been reported.

3. Which biochemical test can be used to differentiate Campylobacter jejuni from other clinically relevant Campylobacter species?
a. Oxidase
b. Catalase
c. Motility
d. Hippurate hydrolysis

Answer: d. C. jejuni is positive for hippurate hydrolysis, while other clinically relevant Campylobacter species are negative. Campylobacter avium is also hippurate positive but is rarely encountered in clinical specimens. Multiple species of Campylobacter are catalase positive, and all Campylobacter species are positive for oxidase and motility.

Citation Whitworth MS, Pence MA. 2019 Closing the Brief Case: Postinfectious glomerulonephritis as an unexpected sequela of drinking raw milk. J Clin Microbiol 57:e01790-18. https://doi.org/10.1128/JCM .01790-18.

Editor Carey-Ann D. Burnham, Washington University School of Medicine Copyright $\odot 2019$ American Society for Microbiology. All Rights Reserved. Address correspondence to Morgan A. Pence, morgan.pence@cookchildrens.org. See https://doi.org/10.1128/JCM.01767-18 in this issue for case presentation and discussion. Published 28 March 2019 


\section{TAKE-HOME POINTS}

- Risk factors for campylobacteriosis include ingestion of raw or undercooked poultry, ingestion of unpasteurized dairy products and untreated water, and exposure to dog and cat feces.

- Campylobacter spp. are fastidious organisms and typically do not grow under routine aerobic or anaerobic culture conditions.

- Campylobacter bacteremia may be missed if only a Gram stain is performed on the positive blood culture broth. For positive blood cultures where no organisms are seen, an acridine orange (AO) stain should be used.

- Many Campylobacter spp. will not grow on Campylobacter agars. Campylobacter selective agars are often selective only for $C$. jejuni and C. coli.

- Ciprofloxacin resistance is $25 \%$ to $40 \%$ for C. jejuni and C. coli, and multidrugresistant isolates have been reported. 